\title{
Assessment of Mitral Valve Reserve Function A Structural Approach to Ischemic Mitral Regurgitation
}

\author{
Lini Dong ${ }^{1}$, Lin Yang ${ }^{1}$, Zhijian $\mathrm{Li}^{1}$, and Xin Wang ${ }^{1}$ \\ ${ }^{1}$ Affiliation not available
}

August 6, 2020

\begin{abstract}
Ischemic mitral regurgitation (IMR) is one of the common complications of coronary heart disease. The primary underlying mechanism is ventricular myopathy rather than disease of the valve itself. The decrease of myocardial blood supply will lead to myocardial damage, which will lead to the left ventricular remodeling, left ventricular enlargement, annular dilation, papillary muscle displacement and limited leaflet activity, resulting in mitral regurgitation. IMR has a certain effect on the prognosis of coronary heart disease, and the incidence rate of IMR has been increasing in recent years. IMR is a complex dynamic process, and it is a great challenge to deal with IMR. For patients with moderate or severe IMR, there are still many challenges and controversies in the choice of surgical methods. This article reviews the pathological process of left ventricular remodeling, the evaluation of IMR, the choice of mitral valve (MV) repair or replacement, and the reserve of MV function. Our review suggests that assessment of MV reserve function may be a predictor of IMR. In the future, assessment of MV reserve function may provide further useful information for evaluating MV function and determining MV repair or replacement in patients with IMR.
\end{abstract}

\section{Introduction}

Coronary atherosclerotic heart disease (CHD) is a common disease in the elderly. IMR is a functional mitral regurgitation secondary to the chronic coronary heart disease and LV remodeling. Up to $60 \%$ of patients with myocardial infarction have IMR ${ }^{[1]}$. The typical reason is that the geometric changes in the left ventricle following myocardial injury impede sufficient coaptation of normal mitral leaflets ${ }^{[2]}$. The MV, as a one-way valve, ensures a certain blood volume circulating from left atrium to left ventricle. The MV apparatus is a complicated structure consisting of anatomic components (leaflets, fibromuscular annulus, chords, papillary muscles and the underlying myocardium). The MV apparatus interact to maintain the MV competent during the cardiac cycle ${ }^{[3]}$. During systole, MV closure includes a dynamic interaction between anatomical and physiological factors (preload, afterload and contractility) to reach the maximum mitral coaptation so as to prevent regurgitation. The intraoperative MV function analysis should start with the quantification of mitral regurgitation (MR) and the diagnosis of related mechanism ${ }^{[4]}$. IMR is a common complication of the left ventricular global or local pathological remodeling caused by acute or chronic coronary artery disease $^{[5]}$. It is a form of systolic incompetence, that is, the consequence of progressive annular dilation or leaflet retraction with gradual reduction and failure of systolic leaflet apposition ${ }^{[2]}$. It often represents the pathological results of increased tethering forces and decreased MV leaflets coaptation ${ }^{[6]}$. Therefore, the degree of apposition serves as a "mitral valve reserve" function that allows the apparatus to sustain further remodeling without overt systolic incompetence ${ }^{[7,8]}$. Ring annuloplasty is usually performed to reduce the annular area, increase the valvular coaptation zone, and reduce the severity of $\mathrm{MR}^{[9]}$. However, the remodeling of the MV apparatus in IMR can be heterogeneous that there may be a variable degree of apposition/reserve along the line of coaptation ${ }^{[10,11]}$. Depending upon the available reserve, the upper limit of the normal mitral annular diameter is MV specific and perhaps region specific within the same MV. 
The appreciation of spatial variation of MV reserve brings into question the utility of normative values of mitral annular diameter routinely used for patient selection for annuloplasty ${ }^{[12,13]}$. The "mitral valve reserve" function is determined by the pathophysiology of the underlying disorder, and an extensive discussion about these changes is beyond the scope of this review. However, a brief introduction of "mitral valve reserve" function is important for surgical decisions making from the intraoperative echocardiographic perspective.

\title{
Intraoperative Mitral Regurgitation Assessment
}

\author{
Quantitative vs Semi-Quantitative Methods
}

The strong correlation between MR and prognosis underscores that the assessment of MR severity is an important part for the decision making in patients with $\operatorname{IMR}^{[14,15]}$. The recently updated guidelines of the American Society of Echocardiography (ASE) recommended an comprehensive method involving quantitative, semi-quantitative and qualitative methods to confirm the degree of secondary MR, which was divided into mild, moderate and severe ${ }^{[15-17]}$. The color Doppler is the most commonly used method for quantifying MR. The effective regurgitant orifice area (EROA), regurgitant volume and fraction can be obtained by evaluating the distal MR jet area/left atrium area ratio, vena contracta width and the proximal isovolumetric surface area (PISA) ${ }^{[16]}$. Hoverer, there are some limitations in using color Doppler to evaluate MR, such as eccentric or multiple or non-holosystolic MR jet, non-circular regurgitant orifice, non-hemispherical PISA, and the influence of load conditions ${ }^{[16]}$.

Three dimensional (3D) imaging can provide supplementary information, which is an adjunct to a comprehensive examination of $2 \mathrm{D}$ transesophageal echocardiography (TEE). Specifically, it enhances the spatial orientation and accuracy of linear measurements, allows synchronous visualization of orthogonal views, and allows the volumetric analysis of cardiac chambers without geometric assumptions. 2D imaging can provide a wide range of methods for the identification of cardiac anatomy, physiology and structures of interest, while 3D TEE is used to obtain specific supplementary quantitative and qualitative information from the interested structures at present ${ }^{[18]}$. It is recommended to use $3 \mathrm{D}$ echocardiography to overcome some of the pitfalls encountered in 2D examination: the 3D image acquisition of MR enables the direct planimetry of the vena contracta (i.e., regurgitant orifice area) and thus optimizes the MR assessment in the case of non-hemispherical PISA and multiple jets ${ }^{[16,19,20]}$.

General Anesthesia (GA) and Mitral Regurgitation

GA has been proved to down grade the severity of MR due to its unloading effect on the $\mathrm{LV}^{[21-23]}$. In addition, the severity of MR may vary as the result of the dynamic nature of intraoperative conditions ${ }^{[4]}$. MR is a valvular pathology affected by multiple variables such as preload, LV contractility, heart rhythm, afterload, GA and positive pressure ventilation ${ }^{[24,25]}$. Alijandro $G$, et al. had done a experiment that a baseline intraoperative TEE examination was performed after GA induction to observe the effects of GA put on the grade of mitral regurgitation. They thought the reduction in MR severity was particularly pronounced when the regurgitation was due to insufficient leaflet coaptation (annular dilatation/ventricular dilatation), but did not seem to decrease when the regurgitation was due to a flail leaflet ${ }^{[26]}$. Regurgitation associated with abnormal MV structure is not significantly influenced by GA induction ${ }^{[24,27,28]}$. Functional MR is shown to be improved with GA when compared with its preoperative severity ${ }^{[29,30]}$. Although the pharmacologic simulation of hemodynamics in awake-state has been improved, the undervaluation of MR under GA has not been completely eliminated ${ }^{[24,31]}$. This is particularly challenging in functional MR cases, because there may be significant inconsistencies between the pre- and intraoperative MR severity assessments ${ }^{[31,32]}$.

\section{Chronic Mitral Regurgitation and Structural Remodeling}

Indices of Remodeling

IMR is a common complication of the LV global or local pathological remodeling caused by acute and chronic coronary artery diseases ${ }^{[2]}$. It often represents the pathological result of increased tethering forces and decreased MV coaptation, which finally leads to IMR ${ }^{[33]}$. IMR is common and seriously affects the prognosis. Even mild IMR can have adverse effects on survival. There is a strong grade relationship between 
IMR severity and survival rate ${ }^{[34]}$. The full closure of the MV leaflets is a balance between two opposing forces: the closing force of the LV contraction and the tethering force of the chordae tendineae (Figure 1 ${ }^{[35]}$. IMR is in a self-perpetuating cycle due to the imbalance caused by either a decrease in the closing force or an increase in the tethering force ${ }^{[36]}$.

IMR occurs when the MV leaflets do not adequately cover the MV orifice in systolic period. Two main mechanisms of IMR are generally accepted: ischemic LV dysfunction and non-ischemic dilated cardiomyopathy. IMR results from LV remodeling, which directly affects the spatial relationship between LV and MV. This deformation finally affects the leaflet coaptation and valve competency. The following mechanisms play roles in the pathophysiology of IMR: 1. PM dysfunction: During systole, PM contraction is important to keep the MV leaflets close in the LV. PM ischemia can lead to hypokinesia and detectable MR ${ }^{[37]}$. The anterolateral PM has a dual blood supply, however, the posteromedial papillary muscle has a solitary blood supply ${ }^{[38]}$. Because of the vascular anatomy of the PM, the posterior PM is more susceptible to ischemia ${ }^{[39]}$. 2. MA function: The MA enlargement and flattening also contributes to the development of IMR. The abnormal MA shape, and/or the loss of the saddle-shape, would result in increase of the leaflet stress and abnormal leaflet remodeling ${ }^{[40]}$. 3. Mechanical coordination of systole: a loss of ventricular mechanical coordination after myocardial infarction would decrease the closing forces during systole, which is thought to be a factor in deteriorating $\mathrm{IMR}^{[41]}$. The disordered contraction of the LV near the PM would increase the tethering forces ${ }^{[42]}$. Dyssynchrony between atrial and ventricular systole would generate diastolic $\mathrm{MR}^{[43]}$. Due to the $\mathrm{MR}$, the time required to reach the maximal coaptation during acute ischemia is prolonged, which would result in severe MR even during "early systole" and maximal coaptation ${ }^{[4]}$.

IMR is believed to initiate from LV remodeling caused by increased diastolic wall stress and persistent increased end-systolic volume ${ }^{[45]}$. The lateral and apical PM displacement secondarily affects the MV coaptation, resulting in the valve incompetence. In IMR, the tethering forces exerted by the chordae are increased while the closing forces are reduced due to LV systolic dysfunction. The PM displacement result from from a regional LV remodeling or the global LV dilation after MI, so one or both PM can be affected. When abnormal wall motion and local remodeling in a specific region lead to adequate MV tethering to generate $\mathrm{IMR}^{[46]}$. MV tethering is symmetric in the global remodeling, while asymmetric tethering mainly occurs following localized LV remodeling and mostly affects the posterior $\mathrm{PM}^{[47]}$.

Intraoperative Application

The presence of IMR has a negative impact on survival rate, and there is a significantly graded relationship between IMR severity and reduced survival. The use of undersized ring annuloplasty for MV repair has become the preferred treatment strategy for $\operatorname{IMR}^{[48,49]}$. Although general consensus has been reached on coronary artery bypass grafting $(\mathrm{CABG})$ and MV surgery for patients with severe MR, clinical dilemma exists in patients with mild to moderate $\mathrm{MR}^{[50]}$. Govindan Set al.reported that there was no significant change in the MV nonplanarity angle in patients with mild or moderate IMR who underwent revascularization alone when they received 2D, 3D TEE and MV assessment before and immediately after the CABG $(\mathrm{n}=20)^{[51]}$. These patients who undergo CABG surgery alone may leave them with obvious residual disease, while the inclusion of MV surgery in the CABG surgery will increase the perioperative risks ${ }^{[2]}$. A growing body of literature indicates an unacceptably high risk of persistent or recurrent IMR after reduction annuloplasty ${ }^{[7,53]}$; however, in patients without recurrent IMR, MV repair may be more beneficial than valve replacement, especially in terms of LV remodeling and function. Reduction annuloplasty is an effective treatment for annular dilatation, but it can make the posterior leaflet move forward and intensify the leaflet tethering ${ }^{[54,55]}$. Vergnat Met al. had made comparisons of 3D TEE data before and after a flat $(\mathrm{n}=9)$ or a saddle annuloplasty $(\mathrm{n}=9)$ was implanted in patients with severe IMR. They found that the shape of the ring affects the curvature of the leaflet. Implantation of a saddle ring kept annular nonplanarity and showed higher 3D leaflet curvature across the MV surface, whereas flat rings reduced annular nonplanarity and flattened leaflet significantly in all but the P1 region ${ }^{[56]}$. This strongly demonstrates that an imaging strategy that can reliably determine the risk of annuloplasty failure and predict recurrence preoperatively would be considered during surgical decision-making, so as to improve the surgical results. 


\section{3、Ischemic Mitral Regurgitation-Repair vs Replacement}

IMR is a result of adverse LV remodeling after myocardial injury, including enlargement of the LV chamber and mitral annulus, apical and lateral displacement of the papillary muscles, leaflet tethering and decreased closing forces. These processes can lead to malcoaptation of the leaflets and varying degrees of MR, which can fluctuate dynamically with heart rhythm, volume status, afterload, and residual ischemia ${ }^{[57]}$. The leaflets are normal, and the pathological changes appear in the myocardium rather than in the valve itself. Therefore, the treatment of functional IMR is quite different from that of primary degenerative $\mathrm{MR}^{[58]}$. Practice guidelines recommend that for patients with severe IMR who experience restrictive symptoms despite the best available medical treatment and possibly cardiac resynchronization, MV repair or chordal-sparing replacement should be considered ${ }^{[59,60]}$. However, these guidelines do not specify whether to do the MV repair or replacement, because there is no clear evidence on which of these intervention is better. Clinical studies have shown that MV repair is associated with lower perioperative mortality ${ }^{[61-63]}$, but replacement provides better long-term correction and lower risk of recurrence (an important consideration is that recurrence of mitral regurgitation may lead to atrial fibrillation, heart failure and readmission) (Table 1$)^{[64-68]}$. But some studies suggest the early mortality of the repair group is higher than that of the replacement group ${ }^{[69,70]}$. And some other studies have demonstrated that survival after combined surgery is mainly affected by factors related to the patient's condition during the operation, but not by the MV repair or replacement (Table 1$)^{[71,72]}$. This perceived trade off between reduced operative morbidity and mortality with repair and better long term IMR correction with replacement has produced significant variation in surgical practice for this high-prevalence condition $^{[58]}$.

\section{Concept of Coaptation Reserve}

Definition

"Coaptation reserve" defined by the actual contact area of the leaflets is less well established, but is still crucial for the intraoperative guidance of MV repair. The leaflet coaptation failure of IMR is due to annular dilatation, leaflet tethering caused by papillary muscle displacement, or both ${ }^{[73]}$. "Mitral valve reserve" refers to the degree of apposition of the MV leaflets that allows the apparatus to maintain further remodeling without overt systolic incompetence ${ }^{[7,8]}$. Restrictive annuloplasty is performed under these circumstances to reduce annular area, provide a greater zone of coaptation between the MV leaflets, and consequently reduce the severity of $\mathrm{MR}^{[9]}$. However, in IMR, the remodeling of the MV apparatus can be heterogeneous with varying degrees of apposition/reserve along the coaptation line ${ }^{[10,11]}$.

\section{Calculation}

The overall and local 3D pathological anatomy of IMR is highly complex and varies widely during patients. All patients with IMR have varying degrees of annular dilatation and leaflet tethering, but the relative contribution of these parameters to valve incompetence differs significantly among patients. This implies that depending upon the available reserve, the upper limit of the normal mitral annular diameter is MV specific and perhaps region specific within the same MV.Mahmood Fet al. had made regional comparisons of 3D TEE data from patients with IMR underwent MV surgery $(n=66)$ and patients with normal valvular and biventricular function $(\mathrm{n}=10)$ to identify measurements that reliably differentiate normal from remodeled MVs. They found that extension of the middle potion of the anterior annulus, larger nonplanarity angle, and increased tenting angle of the posteromedial scallop of the posterior leaflet were sufficient to distinguish IMR from the control group. They thought specific 3D variations in the MV regional geometry can be used to reliably identify a significantly remodeled valve apparatus ${ }^{[74]}$. Cho E Jet al. suggested that MA height likely to be a useful prognostic factor in choosing the timing of surgery in patients with chronic primary MR. Annulus height/BSA can provide supplementary information for predicting the postoperative LA remodeling after successful MV repair ${ }^{[75]}$. Bretschneider $\mathrm{C}$ et al. considered the presence of PM infarction was not associated with IMR, because the severity of mitral regurgitation was not increased compared with patients with partial or no PM infarction ${ }^{[76]}$.

For the unacceptably high risk of persistent or recurrent IMR after reduction annuloplasty, what the "mitral 
valve reserve" can do to predict the recurrence? Gogoladze Get al. had made a experiment that regional coaptation sections were analyzed in cardiac surgery patients with normal MVs ( $\mathrm{n}=10)$ or with functional MR $(n=10)$. They found that the anterior leaflet coaptation length $(C L)$ was greater than posterior leaflet, the functional MR was associated with shorter leaflet CLs, the biggest difference in CLs was in A2-P2, and coaptation depth was higher in the functional MR group. They thought there was a "anterior leaflet reserve"for posterior movement of the coaptation line to compensate for annular dilation and left ventricular enlargement so as to maintain competency until the anterior leaflet CL was insufficient, followed by the functional $\mathrm{MR}^{[77]}$. Wei Det al. had also done a study about the association between the coaptation height of MV and MR. They measured coaptation height of patients underwent annuloplasty for mitral regurgitation $(n=20)$. The results shown that coaptation height had a significant negative correlation with the degree of MR 12 months after operation. They made a point that MV annuloplasty induced the morphologic change of the MV structure. The coaptation height after MV repair may be one of the key factors in regulation of $\mathrm{MR}^{[7]}$. And there were still other researchers wanted to reveal the relationship between the "mitral valve reserve" and the recurrence after mitral annuloplasty. Wijdh-den Hamer I Jet al. performed 2D and 3D TEE on patients underwent undersized annuloplasty due to IMR $(n=50)$. They thought that MV replacement should be strongly considered in patients with a preoperative P3 tethering angle of [?]29.9 ${ }^{\circ}$ (especially when combined with basal aneurysm/dyskinesis) ${ }^{[10]}$.

A growing body of literature has documented an unacceptably high risk of IMR recurrence after reduction annuloplasty, and a growing number of researchers are interested in knowing the role of the "mitral valve reserve" in predicting the recurrence. Some echocardiographic indices derived from 2D TTE - TEE and 3D TEE modeling, have been collected in several studies during the last decade ${ }^{[78-81]}$. The most commonly used cut-offs points for determining the degree of MV tethering and the risk of MV repair failure are as following: anterior leaflet angle $>25^{\circ}$, posterior leaflet angle $>45^{\circ}$, tenting height [?]11 mm, and the tenting area [?]2.5 $\mathrm{cm}^{2[10,14,82]}$. However, all of these cut-offs are obtained from the integrity of MV. For remodeling of the MV apparatus in IMR can be heterogeneous with a variable degree of reserve along the line of coaptation, the upper limit of the MA diameter is MV specific and perhaps region specific within the same MV. Maybe the cut-offs from regional MV are more important in surgical dicision making. This is worthy of further study and discussion.

\section{5、 Future Applications}

In conclusion, "Mitral valve reserve" means the degree of apposition of the MV leaflets that allows the apparatus to sustain further remodeling without overt systolic incompetence. Remodeling of the MV apparatus in IMR can be heterogeneous with a variable degree of apposition/reserve along the line of coaptation. Depending upon the available reserve, the upper limit of the MA diameter is MV specific and perhaps region specific within the same MV. These regional geometric changes can be used to identify important MV apparatus remodeling that may require intervention. We need to further clarify the role of "mitral valve reserve" in patients with IMR. Preoperative echocardiographic assessment should provide the surgeons with information on the pathology and dimensions of the MV apparatus to predict disease recurrence and support the surgical decision making.

\section{References:}

Ferket Bart S, Thourani Vinod H, Voisine Pierre, et al. Cost-effectiveness of coronary artery bypass grafting plus mitral valve repair versus coronary artery bypass grafting alone for moderate ischemic mitral regurgitation. The Journal of Thoracic and Cardiovascular Surgery. 2020; 159 (6): 2230-2240. 2、 Piérard Luc A, Carabello Blase A. Ischaemic mitral regurgitation: pathophysiology, outcomes and the conundrum of treatment. Eur Heart J . 2010; 31(24): 2996-3005. 3、 Mahmood F, Swaminathan M. Systolic anterior motion and mitral valve reserve function: which one should we care about? J Cardiothorac Vasc Anesth . 2010; 24 (5): 885-886. 4、 Mahmood F, Matyal R. A quantitative approach to the intraoperative echocardiographic assessment of the mitral valve for repair.Anesthesia \& Analgesia . 2015; 121 (1): 34-58. 5 、 Goldstein D, Moskowitz A J, Gelijns A C, et al. Two-Year Outcomes of Surgical Treatment of Severe Ischemic Mitral Regurgitation. N Engl J Med . 2016; 374 (4): 344-353. 6、 Shakil O, Jainandunsing JS, Ilic R, et al. 
Ischemic Mitral Regurgitation: An Intraoperative Echocardiographic Perspective. Journal of Cardiothoracic and Vascular Anesthesia. 2013; 27 (3): 573-585. 7、 Wei D, Han J, Zhang H, et al. The correlation between the coaptation height of mitral valve and mitral regurgitation after mitral valve repair. Journal of Cardiothoracic Surgery . 2017; 12 (1): 120. 8、 Guo Y, He Y, Zhang Y, et al. Assessment of the mitral valve coaptation zone with $2 \mathrm{D}$ and $3 \mathrm{D}$ transesophageal echocardiography before and after mitral valve repair. Journal of Thoracic Disease . 2018; 10 (1): 283-290. 9 、 Bonow R. ACC/AHA 2006 guidelines for the management of patients with valvular heart disease : a report of the American College of Cardiology/American Heart Association task force on practice guidelines. Journal of the American College of Cardiology . 2006; 48 (3): 598-675. 10、Wijdhden Hamer I J, Bouma W, Lai E K, et al. The value of preoperative 3-dimensional over 2-dimensional valve analysis in predicting recurrent ischemic mitral regurgitation after mitral annuloplasty. J Thorac Cardiovasc Surg . 2016; 152 (3): 847-859. 11、Bouma W, Lai E K, Levack M M, et al. Preoperative Three-Dimensional Valve Analysis Predicts Recurrent Ischemic Mitral Regurgitation After Mitral Annuloplasty. Annals of Thoracic Surgery . 2015; 101 (2): 567-575. 12、Dwivedi G, Mahadevan G, Jimenez D, et al. Reference values for mitral and tricuspid annular dimensions using two-dimensional echocardiography. Echo Research \& Practice. 2014; 1 (2): 43-50. 13、 Mihăilă S, Muraru D, Piasentini E, et al. Quantitative analysis of mitral annular geometry and function in healthy volunteers using transthoracic three-dimensional echocardiography. Journal of the American Society of Echocardiography . 2014; 27 (8): 846-857. 14 - Vahanian A, Alfieri O, Andreotti F, et al. Guidelines on the management of valvular heart disease (version 2012). Joint task force on the management of valvular heart disease of the European Society of Cardiology (ESC); European Association for Cardio-Thoracic Surgery (EACTS). Eur Heart J . 2012; 33: 2451-2496. 15 - Nishimura RA, Otto CM, Bonow RO, et al. 2017 AHA/ACC Focused Update of the 2014 AHA/ACC Guideline for the Management of Patients With Valvular Heart Disease: A Report of the American College of Cardiology/American Heart Association Task Force on Clinical Practice Guidelines. J Am Coll Cardiol . 2017; 70 (2): 252-289. 16、 Zoghbi WA, Adams D, Bonow RO, et al. Recommendations for Noninvasive Evaluation of Native Valvular Regurgitation: A Report from the American Society of Echocardiography Developed in Collaboration with the Society for Cardiovascular Magnetic Resonance. J Am Soc Echocardiogr . 2017; 30: 303-371. 17. Schroder JN, Williams ML, Hata JA, et al. Impact of mitral valve regurgitation evaluated by intraoperative transesophageal echocardiography on long-term outcomes after coronary artery bypass grafting. Circulation . 2005; 112: I293-1298. 18 - Mahmood F, Jeganathan J, Saraf R, et al. A Practical Approach to an Intraoperative Three-Dimensional Transesophageal Echocardiography Examination. Journal of Cardiothoracic \& Vascular Anesthesia . 2015; 30 (2): 470-490. 19 . Heo R, Son JW, Hartaigh B, et al. Clinical Implications of Three-Dimensional Real-Time Color Doppler Transthoracic Echocardiography in Quantifying Mitral Regurgitation: A Comparison with Conventional Two-Dimensional Methods. J Am Soc Echocardiogr . 2017; 30: 393-403. 20、 Zürcher F, Brugger N, Jahren SE, et al. Quantification of Multiple Mitral Regurgitant Jets: An In Vitro Validation Study Comparing Two- and Three-Dimensional Proximal Isovelocity Surface Area Methods.J Am Soc Echocardiogr . 2017; 30: 511-21. 21. Aklog L, Filsoufi F, Flores KQ, et al. Does coronary artery bypass grafting alone correct moderate ischemic mitral regurgitation? Circulation . 2001; 104: I68-I75. 22、Grewal KS, Malkowski MJ, Piracha AR, et al. Effect of general anesthesia on the severity of mitral regurgitation by transesophageal echocardiography. Am J Cardiol . 2000; 85: 199-203. 23. Chin J H, Lee E H, Choi D K, et al. The effect of depth of anesthesia on the severity of mitral regurgitation as measured by transesophageal echocardiography. $J$ Cardiothorac Vasc Anesth . 2012; 26 (6): 994-998. 24、Sanfilippo F, Johnson C, Bellavia D, et al. Mitral Regurgitation Grading in the Operating Room: A Systematic Review and Meta-analysis Comparing Preoperative and Intraoperative Assessments During Cardiac Surgery. Journal of Cardiothoracic E3 Vascular Anesthesia . 2017; 31 (5): 1681-1691. 25 - Essandoh M. Intraoperative Echocardiographic Assessment of Mitral Valve Area After Degenerative Mitral Valve Repair: A Call for Guidelines or Recommendations. $J$ Cardiothorac Vasc Anesth . 2016; 30 (5): 1364-1368. 26. Gisbert A, Souliã“Re V, Denault A Y, et al. Dynamic quantitative echocardiographic evaluation of mitral regurgitation in the operating department. Journal of the American Society of Echocardiography . 2006; 19 (2): 140-146. 27 、 Shiran A, Merdler A, Ismir E, et al. Intraoperative transesophageal echocardiography using a quantitative dynamic loading test for the evaluation of ischemic mitralregurgitation. J Am Soc Echocardiogr . 2007; 20: 690-697. 28、 Mihalatos D G, Gopal 
A S, Kates R, et al. Intraoperative assessment of mitral regurgitation: role of phenylephrine challenge. $J$ Am Soc Echocardiogr. 2006; 19: 1158-1164. 29、 Bach D S, Deeb G M, Bolling S F. Accuracy of intraoperative transesophageal echocardio- graphy for estimating the severity of functional mitral regurgitation. $A m$ J Cardiol . 1995; 76: 508-512. 30、Khabbaz KR, Mahmood F, Shakil O, et al. Dynamic3-dimensional echocardiographic assessment of mitral annular geometry in patients with functional mitral regurgitation. Ann Thorac Surg . 2013; 95: 105-110. 31 - Essandoh M. Intraoperative Grading of Mitral Regurgitation During General Anesthesia: Is Hemodynamic Matching Useful? J Cardiothorac Vasc Anesth . 2018; 32 (2): e39-e40. 32、Gorman JH 3rd, Jackson BM, Enomoto Y, et al. The effect of regional ischemia on mitral valve annular saddle shape. Ann Thorac Surg. 2004; 77: 544-548. 33、Anyanwu AC, Adams DH. Ischemic mitral regurgitation: recent advances. Curr Treat Options Cardiovasc Med . 2008; 10: 529-537. 34 Trichon BH, Felker GM, Shaw LK, et al. Relation of frequency and severity of mitral regurgitation to survival among patients with left ventricular systolic dysfunction and heart failure. Am J Cardiol . 2003; 91: 538-543. 35 - Silbiger JJ. Mechanistic insights into ischemic mitral regurgitation: Echocardiographic and surgical implications. J Am Soc Echocardiogr . 2011; 24: 707-719. 36 - Agricola E, Oppizzi M, Pisani M, et al. Ischemic mitral regurgitation: Mechanisms and echocardiographic classification.Eur $J$ Echocardiogr . 2008; 9: 207-221. 37 - Timek TA, Dagum P, Lai DT, et al. Tachycardia-induced cardiomyopathy in the ovine heart: Mitral annular dynamic three-dimensional geometry. J Thorac Cardiovasc Surg . 2003; 125: 315-324. 38 - Voci P, Bilotta F, Caretta Q, et al. Papillary muscle perfusion pattern. A hypothesis for ischemic papillary muscle dysfunction.Circulation. 1995; 91:1714-1718. 39、Bouma W, Wijdh-den HamerIJ, Koene BM, et al. Long-term survival after mitral valve surgery for post-myocardial infarction papillary muscle rupture. J Cardiothorac Surg . 2015; 10: 11. 40、Dal-Bianco JP, Beaudoin J, Handschumacher MD, et al.Basic Mechanisms of Mitral Regurgitation. Can J Cardiol . 2014; 30: 971-81. 41 Breithardt OA, Sinha AM, Schwammenthal E, et al. Acuteeffects of cardiac resynchronization therapy on functional mitral regurgitation in advanced systolic heart failure. J Am Coll Cardiol . 2003; 41: 765-770. 42 、 He S, Fontaine AA, Schwammenthal E, et al. Integrated mechanism for functional mitral regurgitation: leaflet restriction versus coapting force: in vitro studies. Circulation . 1997; 16 (96):1826-1834. 43、Appleton CP, Basnight MA, Gonzalez MS. Diastolic mitral regurgitation with atrioventricular conduction abnormalities: Relation of mitral flow velocity to transmitral pressure gradients in conscious dogs. J Am Coll Cardiol . 1991; 18: 843-849. 44 - Glasson JR, Komeda M, Daughters GT, et al. Early systolicmitral leaflet "loitering" during acute ischemic mitral regurgitation.J Thorac Cardiovasc Surg . 1998; 116: 193-205. 45、Spinale F G, Ishihra K, Zile M, et al. Structural basis for changes in left ventricular function and geometry because of chronic mitral regurgitation and after correction of volume overload. J Thorac Cardiovasc Surg .1993; 106: 1147-1157. 46 - Watanabe N, Ogasawara Y, Yamaura Y, et al. Geometric Differences of the Mitral Valve Tenting Between Anterior and Inferior Myocardial Infarction with Significant Ischemic Mitral Regurgitation: Quantitation by Novel Software System with Trans thoracic Real-time Three dimensional Echocardiography. J Am Soc Echocardiogr . 2006; 19: 71-75. 47 - Agricola E, Oppizzi M, Maisano F, et al. Echocardiographic classification of chronic ischemic mitral regurgitation caused by restricted motion according to tethering pattern. Eur J Echocardiogr . 2004; 5: 326-334. 48 - Grossi EA, Goldberg JD, LaPietra A, et al. Ischemic mitral valve reconstruction and replacement: comparison of long-term survival and complications. J Thorac Cardiovasc Surg . 2001; 122: 1107-1124. 49、 Nishimura RA, Otto CM, Bonow RO, et al. 2014 AHA/ACC guideline for the management of patients with valvular heart disease: a report of theAmerican College of Cardiology/ American Heart Association Task Force on Practice Guidelines. Circulation . 2014; 129: e521-e643. 50 - Borger MA, Alam A, Murphy PM, et al. Chronic ischemic mitralregurgitation: Repair, replace or rethink? Ann Thorac Surg . 2006; 81: 1153-1161. 51 Govindan S, Hayward G, Mahmood F, et al. Echocardiographic quantification of mitral valvular response to myocardial revascularization. Annals of Cardiac Anaesthesia . 2013; 16(1): 23. 52、Mallidi HR, Pelletier MP, Lamb J, et al. Late outcomes inpatients with uncorrected mild to moderate mitral regurgitation at the time of isolated coronary artery bypass grafting. J Thorac Cardiovasc Surg . 2004; 127: 636-644. 53、Acker MA, Parides MK, Perrault LP, et al. Mitral-valve repair versus replacement for severe ischemic mitral regurgitation. $N$ Engl $J$ Med . 2014; 370: 23-32. 54 - Bouma W, van der Horst ICC, Wijdh-den Hamer IJ, et al. Chronic ischaemic mitral regurgitation. Current treatment results and new mechanism-based surgical approaches. Eur J Cardiotho- 
rac Surg . 2010; 37: 170-185. 55 - Ciarka A, Braun J, Delgado V, et al. Predictors of mitral regurgitation recurrence in patients with heart failure undergoing mitral valve annuloplasty. Am J Cardiol . 2010; 106: 395-401. 56 - Vergnat M, Mahmood F, Cheung A T, et al. The influence of annuloplasty ring shape on leaflet curvature in human ischemic mitral regurgitation. Journal of the American College of Cardiology . 2010; 55 (10): E1368-E1368. 57 - Acker M A, Parides M K, Perrault L P, et al. Mitral-valve repair versus replacement for severe ischemic mitral regurgitation. New England Journal of Medicine . 2014; 370 (1): 23. 58. Di Salvo TG, Acker MA, Dec GW, et al. Mitral valve surgery in advanced heart failure. J Am Coll Cardiol . 2010; 55: 271-82. 59、Bonow RO, Carabello BA, Kanu C, et al. ACC/AHA 2006 guidelines for the management of patients with valvular heart disease: a report of the American College of Cardiology/ American Heart Association Task Force on Practice Guidelines (writing committee to revise the1998 Guidelines for the Management of Patients with Valvular Heart Disease): developed in collaboration with the Society of Cardiovascular Anesthesiologists: endorsed by the Society for Cardiovascular Angiography and Interventions and the Society of Thoracic Surgeons. Circulation . 2006; 114 (5): e84-e231. 60 - Vahanian Alec, Alfieri Ottavio, Andreotti Felicita, et al. Guidelines on the management of valvular heart disease (version 2012)The Joint Task Force on the Management of Valvular Heart Disease of the European Society of Cardiology (ESC) and the European Association for Cardio-Thoracic Surgery (EACTS). European Heart Journal . 2013; 66 (2): E1-E42. 61 - Akar A R, Durdu S, Zaim C, et al. Clinical outcome and factors affecting surgical decision for repair versus replacement in patients with mitral regurgitation. Anadolu Kardiyoloji Dergisi . 2010; 10 (4): 358-366. 62、Fino C, Iacovoni A, Ferrero P, et al. Determinants of functional capacity after mitral valve annuloplasty or replacement for ischemic mitral regurgitation. Journal of Thoracic \& Cardiovascular Surgery . 2015; 149 (6): 1595-1603. 63、Gillinov A M, Wierup P N, Blackstone E H, et al. Is repair preferable to replacement for ischemic mitral regurgitation? Journal of Thoracic \&3 Cardiovascular Surgery . 2001; 122 (6):1125-1141. 64 - Acker M A, Parides M K, Perrault L P, et al. Mitral-Valve Repair versus Replacement for Severe Ischemic Mitral Regurgitation. New England Journal of Medicine . 2014, 370 (1): 23-32. 65 、 Qiu Z, Xin C, Ming X, et al. Is mitral valve repair superior to replacement for chronic ischemic mitral regurgitation with left ventricular dysfunction? Journal of Cardiothoracic Surgery . 2010; 5(1): 107. 66 - Mihos C G, Santana O. Mitral valve repair for ischemic mitral regurgitation: lessons from the Cardiothoracic Surgical Trials Network randomized study. Journal of Thoracic Disease . 2016; 8 (1): E94. 67、Lorusso R, Gelsomino S, Vizzardi E, et al. Mitral valve repair or replacement for ischemic mitral regurgitation? The Italian Study on the Treatment of Ischemic Mitral Regurgitation (ISTIMIR). The Journal of Thoracic and Cardiovascular Surgery . 2013; 145 (1):128-139. 68 - Goldstein D, Moskowitz A J, Gelijns A C, et al. Two-Year Outcomes of Surgical Treatment of Severe Ischemic Mitral Regurgitation. N Engl J Med . 2016; 374 (4): 344-353. 69 Sadeghian H, Karimi A, Mahmoodian M, et al. Repair Versus Replacement for Ischemic Mitral Regurgitation. Journal of Tehran University Heart Center . 2008; 3 (2): 89-94. 70、 Lio A, Miceli A, Varone E, et al. Mitral valve repair versus replacement in patients with ischaemic mitral regurgitation and depressed ejection fraction: risk factors for early and mid-term mortality. Interact Cardiovasc Thorac Surg . 2014; 19 (1): 64-69. 71 - Maltais S, Schaff H V, Daly R C, et al. Mitral regurgitation surgery in patients with ischemic cardiomyopathy and ischemic mitral regurgitation: factors that influence survival. Journal of Thoracic $\&$ Cardiovascular Surgery . 2011; 142 (5): 995-1001. 72、Shumavets V, Shket A, Janushko A, et al. Repair or replace the mitral valve for severe ischemic mitral regurgitation? Journal of Cardiothoracic Surgery . 2013, 8 (1):12-15. 73、 Cobey F C, Swaminathan M, Phillips-Bute B, et al. Quantitative assessment of mitral valve coaptation using three-dimensional transesophageal echocardiography. Annals of Thoracic Surgery . 2014; 97 (6): 1998-2004. 74、 Mahmood F, Knio Z O, Yeh L, et al. Regional Heterogeneity in the Mitral Valve Apparatus in Patients With Ischemic Mitral Regurgitation.Annals of Thoracic Surgery . 2017; 103 (4): 1171-1177. 75. Cho E J, Park S J, Lee G Y, et al. Effects of Decreased Annular Height and Annular Saddle-Shaped Non-Planarity in Degenerative Severe Mitral Regurgitation with Normal Left Ventricular Ejection Fraction: Real-Time 3D Transesophageal Echocardiography. Journal of Cardiovascular Ultrasound . 2017; 25 (2): 47-56. 76 - Bretschneider C, Heinrich H K, Seeger A, et al. Impact of Papillary Muscle Infarction on Ischemic Mitral Regurgitation Assessed by Magnetic Resonance Imaging. Rofo . 2017; 190 (1): 42-50. 77 - Gogoladze G, Dellis S L, Donnino R, et al. Analysis of the mitral coaptation zone in normal and functional regurgitant valves. Annals of Thoracic Surgery . 2010; 89 (4):1158-1161. 78, Lee 
A P, Acker M, Kubo SH, et al. Mechanisms ofrecurrent functional mitral regurgitation after mitralvalve repair in nonischemic dilated cardiomyopathy: importance of distal anterior leaflet tethering. Circulation . 2009; 119: 2606-2614. 79 、Wijdh-den Hamer IJ, Bouma W, Lai EK, et al. The value of preoperative 3-dimensional over 2-dimensionalvalve analysis in predicting recurrent ischemic mitral regurgitation after mitral annuloplasty. J ThoracCardiovasc Surg . 2016; 152: 847-859. 80 、 Bouma W, Lai EK, Levack MM, et al. Preoperative Three-Dimensional Valve Analysis Predicts Recurrent Ischemic Mitral Regurgitation After Mitral Annuloplasty. Ann Thorac Surg . 2016; 101: 567-75. 81 、 Toida R, Watanabe N, Obase K, et al. PrognosticImplication of Three-Dimensional Mitral Valve Tenting Geometry in Dilated Cardiomyopathy. J Heart Valve Dis . 2015; 24: 577-585. 82、De Bonis M, Al-Attar N, Antunes M, et al. Surgical and interventional management of mitral valve regurgitation: a position statement from the European Society of Cardiology Working Groups on Cardiovascular Surgery and Valvular Heart Disease. Eur Heart J . 2016; 37: 133-9.
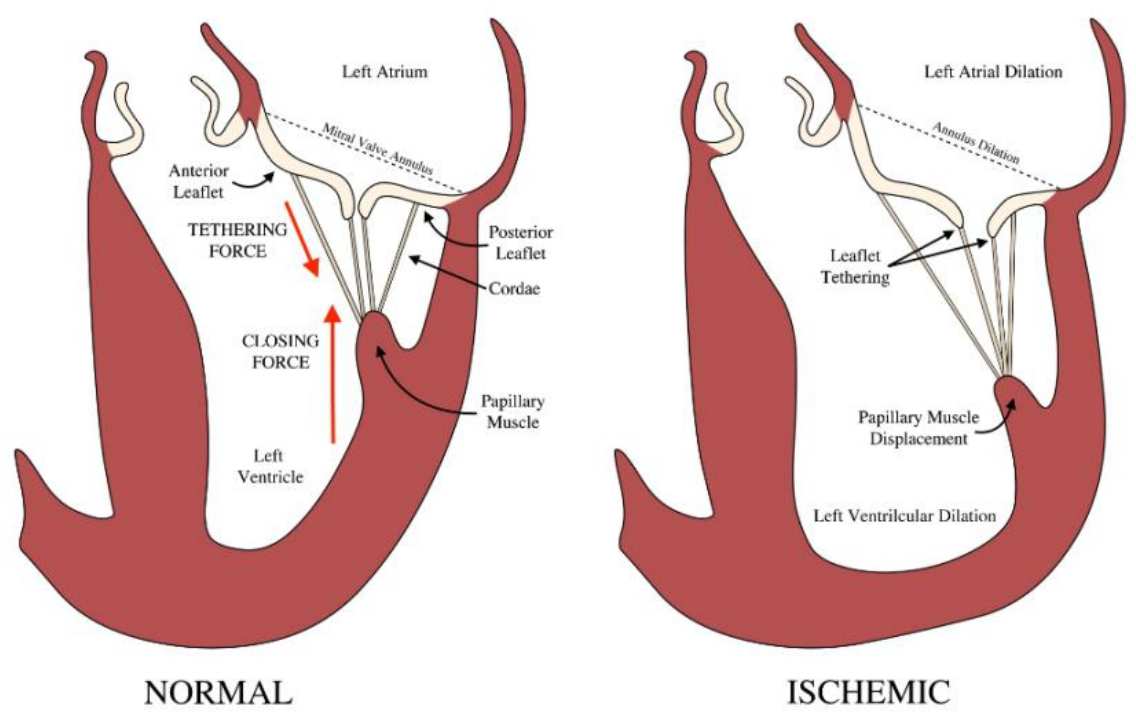

Table 1. Ischemic Mitral Regurgitation-Repair vs Replacement

Study

Hakimeh Sadeghian et al. ${ }^{69}$

Michael A. Acker et al. ${ }^{64}$

Ahmet Rüçhan Akar et al. ${ }^{61}$

Carlo Fino et al. ${ }^{62}$

Zhibing Qiu et al. ${ }^{65}$

A. Marc Gillinov et al. ${ }^{63}$

Simon Maltais et al. ${ }^{71}$

Christos G et al. ${ }^{66}$

Roberto Lorusso et al. ${ }^{67}$

Antonio Lio et al. ${ }^{70}$

D Goldstein et al. ${ }^{68}$

V Shumavets et al. ${ }^{72}$
Summary

Prospective study, CABG plus MV replacement or MV repair were performed in 49 patients Prospective study, in 251 patients with severe IMR, MV repair or chordal-sparing replaceme Retrospective study, 146 patients underwent MV surgery from January, 2004 to January, 20 Retrospective study, 121 patients with significant chronic IMR, who underwent MV repair (1 Retrospective study, 218 patients underwent either MV repair $(\mathrm{n}=112)$ or MV replacement Retrospective study, 482 patients with IMR underwent either valve repair $(\mathrm{n}=397)$ or valve Retrospective study, 387 patients underwent combined CABG and MV surgery, MV repair i Prospective study, 251 patients with severe IMR underwent MV repair $(\mathrm{n}=126)$ or MV repla Retrospective study, from 1996 to 2011, 1006 patients with chronic IMR and LV dysfunction Retrospective study, from July 2002 to February 2011, 126 patients with IMR and LVEF <4 Prospective study, 251 patients with severe IMR underwent MV repair or chordal-sparing re Retrospective study, from 2000 to 2012, 870 patients with coronary artery diseases and signi

IMR: ischemicmitral regurgitation; CABG: coronary artery bypass grafting; LVESVI: left ventricular endsystolic volume index; MV: mitral valve; LVEF: left ventricular ejection fraction. 
Table 2. Studies Describing MV Reserve Function During the Cardiac Cycle in Humans

Study

Gogoladze $\mathrm{G}$ et al. ${ }^{77}$

Cobey F C et al, ${ }^{73}$

Bouma $\mathrm{W}$ et al. ${ }^{80}$

Dan Wei et al. ${ }^{7}$

Cho E J et al. ${ }^{75}$

Feroze Mahmood et al. ${ }^{74}$

Bretschneider $\mathrm{C}$ et al. ${ }^{76}$
Summary

Prospective study, 10 normal;10 with 2 to $4+$ FMR. 3D TEE; data analyzed using QLAB quar Prospective study, 25 patients with FMR underwent cardiac operations. 3D TEE, TomTec Ime Prospective study, 50 patients with IMR. 3D TEE; data analyzed using TomTec Imaging Syste Prospective study, 20 patients underwent MV valvuloplasty for mitral regurgitation were incluc Prospective study, 47 patients with chronic severe MR and preserved LV systolic function sche Prospective study, IMR group, $\mathrm{n}=66$; control group, $\mathrm{n}=10$. 3D TEE; measurements taken usir Prospective study, 48 patients with chronic MI. The Magnetic Resonance Imaging (MRI) protc

FMR: functional mitral regurgitation; 3DTEE: 3-dimensional transesophageal echocardiography; MA: mitral annular; MV: mitral valve; IMR: ischemicmitral regurgitation; MI: myocardial infarction; PM: papillary muscles. 